\title{
BMJ
}

\section{HPV testing on self collected cervicovaginal lavage specimens as screening method for women who do not attend cervical screening: cohort study}

\author{
Murat Gök, research fellow, ${ }^{1}$ Daniëlle A M Heideman, molecular biologist, ${ }^{1}$ Folkert J van Kemenade, surgical \\ pathologist, ${ }^{1}$ Johannes Berkhof, medical statistician, ${ }^{2}$ Lawrence Rozendaal, surgical pathologist, ${ }^{1}$ Johan W M \\ Spruyt, gynaecologist, ${ }^{3}$ Feja Voorhorst, gynaecologist, Jeroen A M Beliën, senior researcher, ${ }^{1}$ Milena Babović, \\ manager of regional screening organisation, ${ }^{4}$ Peter J F Snijders, medical biologist , ${ }^{1}$ Chris J L M Meijer, surgical \\ pathologist ${ }^{1}$
}

${ }^{1}$ Department of Pathology, VU University Medical Center, PO Box 7057, 1007

MB Amsterdam, Netherlands

${ }^{2}$ Epidemiology and Biostatistics,

VU University Medical Center, Amsterdam

${ }^{3}$ Obstetrics and Gynaecology, VU University Medical Center,

Amsterdam

${ }^{4}$ Regional Screening Organisation, Hoogoorddreef 54e 1101 BE Amsterdam, Netherlands

Correspondence to: C J L M Meijer cjlm.meijer@vumc.nl

Cite this as: BMJ 2010;340:c1040 doi:10.1136/bmi.c1040

\section{ABSTRACT}

Objective To determine whether offering self sampling of cervicovaginal material for high risk human papillomavirus (HPV) testing is an effective screening method for women who do not attend regular cervical screening programmes.

Design Cohort study (the PROHTECT trial).

Settings Noord-Holland and Flevoland regions of the Netherlands, December 2006 to December 2007, including 13 laboratories, gynaecologists, and more than 800 general practitioners.

Participants 28073 women who had not responded to two invitations to the regular cervical screening programme: 27792 women were assigned to the self sampling group and invited to submit a self collected cervicovaginal sample for HPV testing; 281 were assigned to the recall control group and received a second reinvitation for conventional cytology.

Intervention Women with a positive result on the high risk HPV test on their self sample material were referred to their general practitioner. Women with abnormal results on cytology were referred for colposcopy. Women with normal results on cytology were re-evaluated after one year by cytology and high risk HPV testing and referred for colposcopy if either result was positive.

Main outcome measures Attendance rate in both groups and yield of cervical intraepithelial neoplasia grade II/III or worse ( $\geq$ CIN II/ $\geq$ CIN III) in self sampling responders.

Results The compliance rate in the self sampling group was significantly higher than in the control group (crude $26.6 \%$ v $16.4 \%$, P $<0.001$; adjusted $27.5 \%$ v $16.6 \%$, $\mathrm{P}<0.001)$. The number of detected $\geq \mathrm{CIN}$ II and $\geq \mathrm{CIN}$ III lesions in self sampling responders was 99 (1.3\%) and 76 (1.0\%), respectively. Self sampling responders who had not participated in the previous round of screening (43\%) had increased relative risks of $\geq$ CIN II $(2.04,95 \%$ confidence interval 1.27 to 3.28$)$ and $\geq \operatorname{CIN~III~}(2.28,1.31$ to 3.96 ) compared with self sampling women who had been screened in the previous round (57\%).
Conclusions Offering self sampling by sending a device for collecting cervicovaginal specimens for high risk HPV testing to women who did not attend regular screening is a feasible and effective method of increasing coverage in a screening programme. The response rate and the yield of high grade lesions support implementation of this method for such women.

Trial registration ISRCTN45527158.

\section{INTRODUCTION}

The introduction of organised cervical cancer screening programmes in Western countries has contributed to a decrease in incidence of and mortality from cervical cancer. Nevertheless, one major problem concerning the effectiveness of current cervical screening programmes remains non-attendance. ${ }^{1-4}$

Non-participating women (that is, non-attendees) are at increased risk of cervical cancer. ${ }^{56}$ Therefore, targeting non-attendees is important in achieving optimal protection from screening programmes. Offering self sampling of cervicovaginal material for screening has been suggested as a way of increasing screening compliance. $^{178}$

Cytomorphological evaluation of self sampled cervicovaginal specimens for detection of high grade cervical lesions has been shown to be inferior compared with cervical samples obtained by a physician. Conversely, high risk HPV testing on self collected cervicovaginal samples had at least similar sensitivity for cervical intraepithelial neoplasia grades II/III or worse $(\geq \mathrm{CIN}$ II/ $\geq$ CIN III) compared with cytological reading of a corresponding cervical sample collected by a physician. $^{9-12}$

\section{METHODS}

Patients and procedures

PROHTECT (protection by offering HPV testing on cervicovaginal specimens trial) is a cohort study within the setting of the Dutch population based cervical screening programme to assess the feasibility and 
efficacy of offering cervicovaginal lavage self sampling for high risk HPV testing to women who do not attend the regular screening programme. In the Dutch screening programme, women aged 30-60 are invited once every five years. Non-attendees living in the counties of Noord-Holland or Flevoland $(\mathrm{n}=28073$ ) who had received their screening invitation in 2005 were selected from the regional health council registry. In the screening programme women are asked to make an appointment to have a smear taken by their general practitioner or assistant. Opportunistic smears are disregarded but results are registered in the nationwide pathology database (PALGA). There is no charge for women participating in primary cervical screening, but the costs for the necessary follow-up in cases of abnormal cytology can be recovered from health insurance companies. A "non-attendee" was defined as a woman who neither responded to the regular invitation nor to a standard reminder after six months. Women with previous hysterectomy were excluded.

With a computerised random number generator non-attendees were assigned in a 99:1 ratio to either receive a kit (Delphi Screener (previous PantarheiScreener/Mermaid)) to collect cervicovaginal material for subsequent testing for high risk HPV hybrid capture II (self sampling group, $\mathrm{n}=27$ 792) or to receive a second recall for conventional cytology (recall control group, $\mathrm{n}=281$ ). The Delphi Screener is a lavage device, designed to rinse the upper vagina and cervix and, in combination with HPV testing, has been shown to allow detection of similar high grade yields of CIN to those achieved in cervical smears collected by physicians. $^{9}$ The skewed ratio ensured adequate power to detect a higher attendance rate in the self sampling versus second recall arm, while at the same time maximising the $\geq$ CIN II/ $\geq$ CIN III rate among self sampling responders. The pre-randomised self sampling and recall cohorts were recruited from December 2006 to April 2007, and women were invited to respond within six weeks after the mailing. Only responses received up to December 2007 were scored for analysis.

All invited women were written to at their home address the week before to give notice of receipt of a second recall letter (recall control group) or a self sample kit (self sampling group). Women in the recall control group received an official second reminder to visit their general practitioner for conventional cytology, an explanatory letter, and an informed consent form. Women in the self sampling group received a self sample kit with an explanatory letter, ${ }^{9}$ a collection tube, instructions (written and drawn), an informed consent form, and a return box with the address of the testing laboratory. A telephone helpline and website providing information on the trial (www.hpvthuistest.nl) was available throughout the study.

\section{HPV testing and follow-up algorithm}

Women in the self sampling group were asked to send the collection tube containing their cervicovaginal lavage specimen with the signed informed consent form to the laboratory for high risk HPV testing. Each specimen was tested with the hybrid capture II high risk HPV DNA method at the laboratory. The results of the confirmatory HPV samples taken by general practitioner (see below) were also based on the hybrid capture II tests. On arrival in the laboratory, lavage specimens were concentrated by spinning down, removing all supernatant, and resuspending the pellet in universal collection medium. If no clear cell pellets were visible, samples were considered invalid for testing. In such cases, the woman was sent a second kit to repeat the self sampling at home. Valid samples were subjected to the hybrid capture II high risk HPV DNA test in an automated format on a rapid capture system), according to the manufacturer's instructions (Qiagen, Gaithersburg, MD, USA). This test is designed to detect high risk HPV types 16,18 , $31,33,35,39,45,51,52,56,58,59$, and 68 . Results were expressed as relative light units per cut-off value (RLU/CO). Women with a positive result (RLU/CO $\geq 1$ ) were instructed to visit their general practitioner for their doctor to take a cervical sample for cytology and confirmatory HPV testing in the regional laboratory serving the general practitioner. Women with abnormal cytology results (threshold borderline or mild dyskaryosis) were referred for colposcopy. Cytology of cervical smears taken by the physician was carried out in local laboratories, and results were reported according to the CISOE-A classification, the standard classification system for cytology in the Netherlands, which can easily be translated into the Bethesda classification. ${ }^{10}$ In brief, on the basis of either squamous or columnar abnormalities, cytology results are categorised into three groups (normal, borderline or mild dyskaryosis, and moderate dyskaryosis or worse). Borderline or mild dyskaryosis corresponds in the Bethesda classification to ASCUS $\backslash$ LSIL. Endometrial abnormalities were excluded.

Independent of the HPV test result on the specimen taken by the physician, women with normal cytology results received advice for repeat testing (cytology and HPV testing) after a year and were referred for colposcopy in case of a positive HPV or cytology test result (threshold $\geq$ borderline or mild dyskaryosis). Women with negative results on both tests were referred back to the national screening programme as their risk of a clinically relevant lesion was considered too low to warrant referral for colposcopy. ${ }^{11}$ Colposcopy directed biopsies were taken for histological examination from suspected areas on the cervix according to standard procedures in the Netherlands. ${ }^{12}{ }^{13}$ Histological examination was done in local pathology laboratories and specimens were classified as CIN 0 (that is, within normal limits or including any non-neoplastic lesion such as inflammation, cyst, etc), I, II, or III, or as invasive cancer, according to international criteria. ${ }^{14}{ }^{15} \mathrm{On}$ the basis of biopsy results, women were treated in accordance with the guidelines of the national screening programme. ${ }^{16}$ Based on the cytology results, participating women in the recall control group were 
managed according to the guidelines of the national screening programme. ${ }^{17}$

\section{Statistical analysis}

Power calculation

For the power calculation, we estimated the population of non-attendees in the study area to be 45000 in the year 2005 . We further assumed $10 \%$ of these women would not be eligible and that the response rate in the self sampling group would be $15 \%$ higher than in the recall control group. The power calculation was based on our previous pilot, ${ }^{1}$ which showed compliance rates of $17.6 \%$ and $31.5 \%$ in the recall control and self sampling groups, respectively (two sided $t$ test; $\alpha$ 0.05; 99:1 randomisation; power 0.99999). We chose a 99:1 randomisation to provide sufficient power to detect differences in compliance and to maximise the yield of $\geq$ CIN II/ $\geq$ CIN III in the self sampling cohort.

\section{Response rate}

All trial data were managed within a customised database. The self sampling response was counted on the basis of receipt of informed consent forms plus self samples, and the response of the recall control group was counted on the basis of receipt of informed consent forms as well as through the nationwide network and registry of histopathology and cytopathology in the Netherlands (PALGA; Bunnik, Netherlands). For computing the response rate we included all women who responded actively within 12 months after their invitation to participate in this trial and compared response rates of the groups with $\chi^{2}$ test. In addition to the crude response rates, we also compared the response rates adjusted for women who during the study reported they were not eligible because of previous hysterectomy.

\section{HPV prevalence and yield of high grade CIN and cervical cancer}

Only self sampling material sent in within a year after the invitation was included in HPV prevalence analysis. The 18 month cumulative yields of $\geq$ CIN II and $\geq$ CIN III in women in the self sampling group who submitted a specimen were obtained through the PALGA database. If necessary, physicians were contacted directly for additional clinical data.

\section{Assessment of screening history self sampling responders}

Programmed screening in the Netherlands involves seven cytology invitations during a lifetime to women aged 30-60 - that is, every five years. The PALGA database provided the screening history of attendees in the two groups. ${ }^{18}$ In analysing the screening history among the women who had been invited for a previous round of screening (that is, those aged $\geq 34$ ), women were considered to have missed the previous screening round if a smear sample had not been taken within the past seven years.

Risks of $\geq$ CIN II and $\geq$ CIN III were calculated for women aged $\geq 34$ who did not participate in the previous round relative to those who were screened in the previous round. The relative risks were adjusted for age by the Mantel-Haenszel method, with age stratified at 34-38, 39-43, 44-48, 49-53, 54-58, and $\geq 59$. Women aged $\leq 33$ could not have a screening history because of their age and were excluded. The association between the relative risks and age, as well as between the relative risks and screening history, were tested by the Mantel-Haenszel test of homogeneity.

\section{RESULTS}

\section{Characteristics of trial cohort}

Figures 1 and 2 show the flow of women through the study. The public health database indicated that 28073 women in the 2005 cohort who received an invitation to screening in the study area were registered as nonattendees, instead of the expected 45000 . Of these, 27792 were allocated to the self sampling arm and 281 to the recall control arm. During the study 906 women $(3.3 \%)$ in the self sampling group and four women $(1.4 \%)$ in the recall control group reported having had a hysterectomy, leaving 26886 eligible women in the self sampling group and 277 in the recall control group. There were no significant differences between the age distributions in both arms.

\section{Participation rate}

In the self sampling group, 7404 of 27792 (26.6\%) women sent a self sampled specimen for HPV testing and $51(0.2 \%)$ decided to visit their general practitioner for conventional cervical cytology. When we adjusted for women who were not eligible because of hysterectomy, the percentage of women who responded by submitting a self sample was $27.5 \%$. The self sampling response rate did not vary with age (Pearson $\chi^{2}=7.15$, $\mathrm{df}=6 ; \mathrm{P}=0.307)$.

In the recall control group, 46 of 281 (16.4\%) women visited their general practitioner for cervical cytology. After adjustment for non-eligibility, the response rate was $16.6 \%$ (46/277). Taken together, the difference in compliance rates between the groups was $10.2 \%$

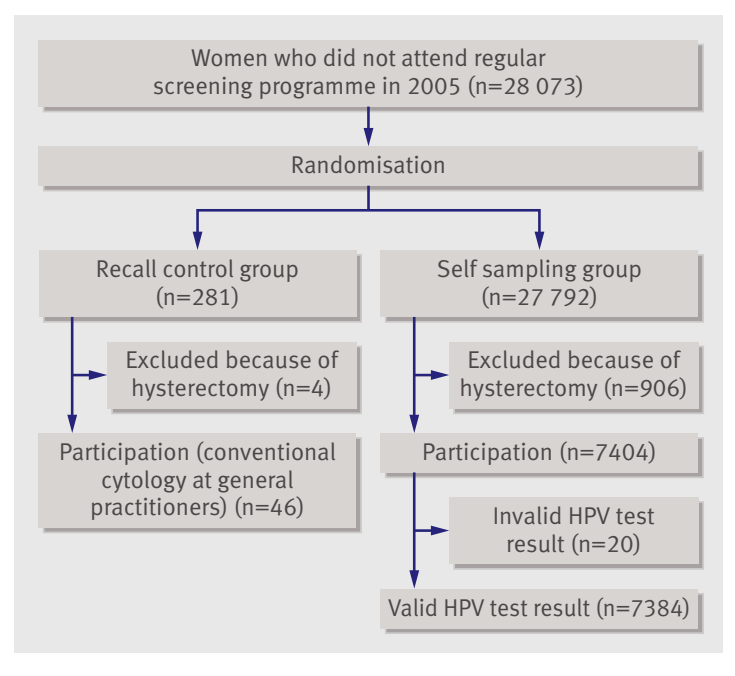

Fig 1| Study design for comparison of compliance rates between recall control group and self sampling group 


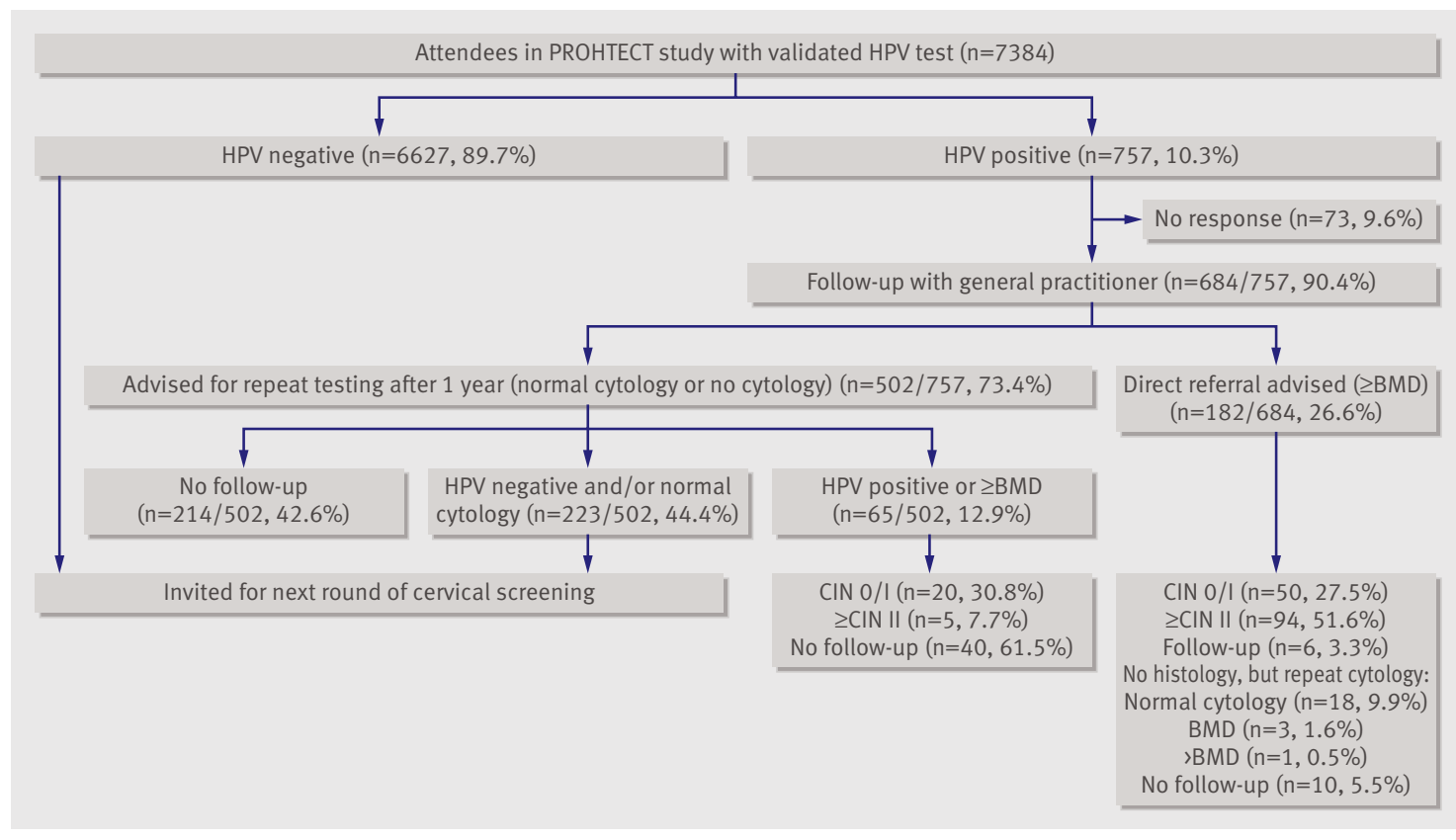

Fig 2 | Study design for evaluation of yield of $\geq$ CIN II in women of self sampling group (BMD=borderline or mild dyskaryosis)

$(26.6 \%$ v $16.4 \%, 95 \%$ confidence interval $5.9 \%$ to $14.6 \%, \mathrm{P}<0.001)$ or $10.9 \%(27.5 \%$ v $16.6 \%, 6.5 \%$ to $15.3 \%, \mathrm{P}<0.001)$ after adjustment for those who were not eligible.

To evaluate whether screening history had an effect on participation in women allocated to self sampling, we compared the screening history of women in the recall control group $(n=277)$ with women in the self sampling group $(\mathrm{n}=26886)$. When we excluded women aged $<33$ because they could not have a screening history (49 in the recall control group and 3398 in the self sampling group), 228 eligible women were left in the recall control group and 22988 in the self sampling group. There were 34 and 6227 attendees aged $\geq 34$, respectively. Of the 228 women in the recall control group, $61(26.8 \%)$ had had a smear taken in the past seven years, compared with $5967(26.0 \%)$ of the 22988 women in the self sampling group, indicating no significant differences.

We examined the effect of having no smear in the past seven years on attendance of women in the recall control group compared with the effect on attendance in the self sampling group. In the recall control group $9.0 \%(15 / 167)$ of the women responded to recall compared with $15.8 \%(2694 / 17021)$ of the women in the self sampling group. Thus, the relative risk for participation of women without a smear in the past seven years in the self sampling group was 1.76 (1.09 to 2.86) compared with women who had no smear in the past seven years in the recall control group $(\mathrm{P}=0.016)$.

For the women who had had a smear in the past seven years, these proportions were 19/61 (31.1\%) for the recall control group and 3533/5967 (59.2\%) for the self sampling group, resulting in a relative risk of participation in the self sampling group of $1.90(1.31$ to $2.76, \mathrm{P}<0.001)$. As the relative risks of participation of women who had or had not had a smear in the past seven years did not differ $(1.90 v 1.76, \mathrm{P}=0.80)$, it seems unlikely that screening history introduced bias in the participation of women in self sampling in both groups.

\section{HPV detection rate self sampling attendees}

Of the 7404 women who submitted a self collected sample, $7384(99.8 \%)$ had a valid hybrid capture II test result (fig 1) and $757(10.3 \%)$ were positive for high risk HPV (fig 2). The percentage positive for HPV decreased with age $(t=-6.77 ; \mathrm{P}<0.001)$ until age $39-43$, when a plateau was reached $(t=-0.30 ; \mathrm{P}=0.77)$.

Women who had positive results for high risk HPV were advised to visit their general practitioner for both conventional cytology and a second high risk HPV test on a cervical sample collected by the physician. A valid HPV result was recorded for 491, and 288 (58.7\%) were positive for cervical HPV. Of the 203 with a negative result, $81(39.9 \%)$ originally displayed hybrid capture II RLU/CO values $<2$ on the self collected sample. Conversely, only $21(7.3 \%)$ with a positive result had a $\mathrm{RLU} / \mathrm{CO}$ value $<2$ on the self sample.

\section{Compliance with follow-up}

Of the self sampling attendees with positive HPV results, 90.4\% (684/757) complied with follow-up with their general practitioner. Of these 684, 437 $(63.9 \%)$ followed the trial protocol and had both cytology and HPV test results, whereas $61(8.9 \%)$ had only an HPV test and $186(27.2 \%)$ had only a cytology test. Thus 623/757 (82\%) women had cytological followup. A total of $182(26.6 \%)$ women had abnormal results on cytology, of whom $150(82.4 \%)$ adhered to the direct referral advice for colposcopy. The 502 remaining women $(73.4 \%)$ were advised have a repeat testing 
Table 1 Yields of $\geq \mathrm{CIN} \mathrm{II/III} \mathrm{in} \mathrm{women} \mathrm{who} \mathrm{carried} \mathrm{out}$ cervical self sampling (categorised by age)

\begin{tabular}{|c|c|c|c|}
\hline Age (years) & No of women & $\begin{array}{c}\text { No (\%) with } \\
\geq \text { CIN II }\end{array}$ & $\begin{array}{c}\text { No (\%) with } \\
\geq \text { CIN III }\end{array}$ \\
\hline$\leq 33$ & 1157 & $29(2.5)$ & $23(2.0)$ \\
\hline $34-38$ & 1497 & $30 *(2.0)$ & $23^{\star}(1.5)$ \\
\hline $39-43$ & 1266 & $10^{\star}(0.8)$ & $6^{\star}(0.5)$ \\
\hline $44-48$ & 1139 & $11^{\star}(1.0)$ & $8^{\star}(0.7)$ \\
\hline 49-53 & 918 & $9 *(1.0)$ & $7 *(0.8)$ \\
\hline $54-58$ & 825 & $6(0.7)$ & $5(0.6)$ \\
\hline$\geq 59$ & 582 & $4^{\star}(0.7)$ & $4^{\star}(0.7)$ \\
\hline Total & 7384 & $99+(1.3)$ & 76† (1.0) \\
\hline $\begin{array}{l}\text { Total (excluding } \\
\text { age } \leq 33 \text { ) }\end{array}$ & 6227 & $70 \dagger(1.1)$ & $53+(0.9)$ \\
\hline
\end{tabular}

after a year on a sample collected by their general practitioner, and $287(57.2 \%)$ complied. Based on a positive high risk HPV result or cytology result, or both, 65 $(12.9 \%)$ women were advised to undergo colposcopy and $25(38.5 \%)$ of them did so.

\section{Yield of high grade CIN and cervical cancer}

Among the 150 women with $\geq$ borderline or mild dyskaryosis who visited a gynaecologist at baseline, 94 $\geq$ CIN II lesions were detected, including five invasive carcinomas. Furthermore, five $\geq$ CIN II lesions were detected among the 25 women who complied with the referral for colposcopy after repeat testing at one year. The cumulative 18 month yields of $\geq$ CIN II and $\geq$ CIN III in women with a positive HPV self sampling test were $1.3 \%(99 / 7384)$ and $1.0 \%(76 / 7384)$, respectively (table 1).

Yield of high grade CIN and cervical cancer in relation to screening history

Among women aged $\geq 34$, those who did not have a cervical smear taken at the previous round had more $\geq \mathrm{CIN}$ II (relative risk 2.04, 1.27 to $3.28, \mathrm{P}=0.003$; table 2$)$ and more $\geq$ CIN III $(2.28,1.31$ to 3.96 , $\mathrm{P}=0.003$; table 3 ) than women who did have a cervical smear at the previous round. This association between screening history and cervical intraepithelial neoplasia was not related to age ( $\mathrm{P}$ values Mantel-Haenszel test of homogeneity 0.639 for $\geq \mathrm{CIN}$ II and 0.515 for $\geq \mathrm{CIN}$ III). All five carcinomas were detected in women aged $\geq 34$ who had not been screened at the previous round.

\section{DISCUSSION}

In the Netherlands in 2005, $65 \%$ of women attended the cervical screening programme (annual report of the regular screening programme, 2006, www.bevolking sonderzoek.info/). By offering self sampling to nonattendees, and taking into account the 18\% loss of cytology in the follow-up in this group, the real effect on attendance in the screening programme would be an extra $5.2 \%\left(6.3 \%(27.5 \% \text { of } 23 \%)^{*}(100 \%-18 \%)\right)$. The total attendance in the screening programme would then increase to $70.2 \%(5.2 \%+65 \%)$. Moreover, we showed that the cumulative incidence of $\geq$ CIN II yield in our study was $1.3 \%(99 / 7384)$, while the CIN lesions found via regular screening programme in 2005 was $0.8 \%$ (data received from PALGA).

\section{Screening history of non-attendees}

The finding of a twofold and more than twofold relative risk of $\geq \mathrm{CIN}$ II and $\geq \mathrm{CIN}$ III, respectively, in self sampling women aged $\geq 34$ who did not attend the previous screening round is in line with the assumption that background risk for $\geq \mathrm{CIN} \mathrm{II/} \geq \mathrm{CIN}$ III is increased after women miss one screening round. In the self sampling group, the association between screening history and CIN was independent of age $(\mathrm{P}=0.639$ for $\geq \mathrm{CIN}$ II and 0.515 for $\geq$ CIN III).

\section{Strengths and limitations}

We did not include a recall control group for comparison of yield of $\geq$ CIN II/ $\geq$ CIN III with the self sampling group because data from our previous work indicated that non-attendees of the regular screening programme respond poorly to any repeat invitation letter. ${ }^{1}$ Instead, we used a randomisation ratio of self sampling versus recall women in favour of maximising detection of $\geq$ CIN II/ $\geq$ CIN III in the self sampling group to allow an accurate assessment of the yield achieved by self sampling combined with HPV testing in non-attendees.

Table 2 |Yield and risk of $\geq$ CIN II in women aged $\geq 34$ in relation to participation in previous round of screening (categorised by age)

\begin{tabular}{|c|c|c|c|c|c|c|}
\hline \multirow[b]{2}{*}{ Age (years) } & \multicolumn{2}{|c|}{ Screened in previous round } & \multicolumn{2}{|c|}{$\begin{array}{l}\text { Not screened in previous } \\
\text { round }\end{array}$} & \multirow{2}{*}{$\begin{array}{l}\text { Rate of participation in } \\
\text { previous round }(95 \% \mathrm{Cl})\end{array}$} & \multirow{2}{*}{$\begin{array}{c}\text { Relative risk }(95 \% \mathrm{Cl}) \text { of } \\
\geq \mathrm{CIN} \mathrm{II}\end{array}$} \\
\hline & No of women & $\geq \mathrm{CIN}$ II & No of women & $\geq$ CIN II & & \\
\hline $34-38$ & 809 & 13 & 688 & $17^{\star}$ & $54.0(51.5$ to 56.6$)$ & $1.54(0.75$ to 3.14$)$ \\
\hline $39-43$ & 721 & 4 & 545 & $6^{*}$ & $57.0(54.2$ to 59.7$)$ & $1.98(0.56$ to 7.00$)$ \\
\hline $44-48$ & 684 & 3 & 455 & $8^{\star}$ & 60.1 (57.2 to 62.9$)$ & 4.01 (1.07 to 15.03$)$ \\
\hline $49-53$ & 531 & 5 & 387 & $4^{*}$ & $57.8(54.7$ to 61.0$)$ & $1.10(0.30$ to 4.06$)$ \\
\hline $54-58$ & 463 & 2 & 362 & 4 & 56.1 (52.7 to 59.5$)$ & $2.56(0.47$ to 13.89$)$ \\
\hline$\geq 59$ & 325 & - & 257 & $4^{\star}$ & 55.8 (51.8 to 59.9$)$ & - \\
\hline Total & 3533 & 27 & 2694 & $43 \dagger$ & 56.7 (55.5 to 58.0$)$ & 2.04 (1.26 to 3.28$)$ \\
\hline
\end{tabular}


Table $3 \mid$ Yield and risk of $\geq$ CIN III in women aged $\geq 34$ in relation to participation in previous round of screening (categorised by age)

\begin{tabular}{|c|c|c|c|c|c|c|}
\hline \multirow[b]{2}{*}{ Age (years) } & \multicolumn{2}{|c|}{ Screened in previous round } & \multicolumn{2}{|c|}{$\begin{array}{l}\text { Not screened in previous } \\
\text { round }\end{array}$} & \multirow{2}{*}{$\begin{array}{l}\text { Rate of participation in } \\
\text { previous round }(95 \% \mathrm{Cl})\end{array}$} & \multirow{2}{*}{$\begin{array}{c}\text { Relative risk }(95 \% \mathrm{Cl}) \text { of } \\
\geq \mathrm{CIN} \text { III }\end{array}$} \\
\hline & No of women & $\geq$ CIN III & No of women & $\geq$ CIN III & & \\
\hline $34-38$ & 809 & 11 & 688 & $12^{\star}$ & $54.0(51.5$ to 56.6$)$ & 1.28 (0.57 to 2.89$)$ \\
\hline $39-43$ & 721 & 2 & 545 & $4^{\star}$ & $57.0(54.2$ to 59.7$)$ & 2.65 (0.49 to 14.39$)$ \\
\hline $44-48$ & 684 & 2 & 455 & $6^{*}$ & 60.1 (57.2 to 62.9) & 4.51 (0.91 to 22.25$)$ \\
\hline $49-53$ & 531 & 3 & 387 & $4^{*}$ & $57.8(54.7$ to 61.0$)$ & $1.83(0.41$ to 8.13$)$ \\
\hline $54-58$ & 463 & 1 & 362 & 4 & 56.1 (52.7 to 59.5$)$ & $5.12(0.57$ to 45.57$)$ \\
\hline$\geq 59$ & 325 & - & 257 & $4^{*}$ & 55.8 (51.8 to 59.9$)$ & - \\
\hline Total & 3533 & 19 & 2694 & $34 \dagger$ & 56.7 (55.5 to 58.0$)$ & 2.28 (1.31 to 3.96$)$ \\
\hline
\end{tabular}

*Including one carcinoma.

†Including five carcinomas.

A potential bias in our attendance data could be that, unlike responders to a re-invitation for cytology, self sampling responders might have been more likely to respond for curiosity reasons, despite already being opportunistically screened before the study invitation. To address this we analysed the effect of screening history on participation via self sampling versus a second recall. When we took into account the screening history of women responders aged $\geq 34$ in both arms, there was no indication that previously screened women would have a relatively higher preference for self sampling than women who were not screened within the past seven years.

Interestingly, self sampling responders showed high adherence to direct follow-up regimens, both at the general practitioner level $(90.4 \%)$ and at the level of direct referral for colposcopy $(94.5 \%)$. In size the latter is comparable with follow-up compliance of attendees of the Dutch screening programme (91\%). ${ }^{16}$ Compliance after repeat testing advice $(59 \%$ of women attended after one year), however, was markedly lower than observed in regular screening attendees with similar advice (86\%). ${ }^{19}$ This rather low return rate might be influenced by the fact that most of these women had previous normal cytology test results after a smear taken by a physician.

In $41 \%$ of the women who had an HPV test on both self and physician collected samples, a positive result for high risk HPV in the self sample could not be confirmed in the sample taken by the physician. Most of these discrepant test results were found in women with low hybrid capture II RLU/CO values. In self sampled specimens more HPV infections of vaginal origin, including those of low risk HPV types, might be detected by hybrid capture II. ${ }^{20-25}$ Even with a cut-off level of a positive result on hybrid capture II increased to $\mathrm{RLU} / \mathrm{CO} \geq 2$, there are still discrepancies between positive results for HPV in the self sampled specimens and smears taken by the general practitioner. In that case the total number of HPV positive cases would decrease from 757 to 627 , but we would miss six $\geq$ CIN II lesions (two CIN II, and four $\geq$ CIN III).

Interestingly, the yields of $\geq$ CIN II and $\geq$ CIN III in self sampling responders who attended the previous round and the yields in regular screening responders of the same age tested for high risk HPV by general primer $5+/ 6+$ polymerase chain reaction $(\mathrm{GP} 5+/ 6+$ PCR) were identical $(0.8 \%$ and $0.5 \%$, respectively). ${ }^{2627}$ This strongly suggests that the $\geq \mathrm{CIN}$ II/ $\geq$ CIN III sensitivity of HPV testing in self sampled cervicovaginal material is not inferior to that of HPV testing on smears taken by a physician. This is in agreement with a recent meta-analysis that indicated that self sampling is as sensitive as physician obtained sampling to detect high risk HPV. ${ }^{25}$ Collectively, our data show that targeted efforts should be made to screen self sampling non-attendees who missed a previous screening round, given their increased risk of clinically relevant cervical disease.

We have also shown that the chosen triage algorithm of a cytology test on a conventional smear after an HPV positive self sample is successful. A substantial subset of the $10 \%$ self sampling women who were positive for high risk HPV, however, seemed to have negative cytology results and be negative for high risk HPV at follow-up, which in practice resulted in a marked number of unnecessary visits to the general practitioner for these women. Therefore, alternative triage tools applicable to self sampled material should be considered to prevent redundant sampling by general practitioners. In this context, molecular methylation markers, ${ }^{28}$ which are currently being investigated, are highly promising when applied to self sampled specimens. Furthermore, efforts are ongoing to improve liquid based cytological preparations of cervicovaginal lavage fluids for detecting abnormal cells.

\section{Conclusions}

It is feasible and effective to offer women who do not attend regular cervical screening programmes the choice of self sampling by sending a device for collecting cervicovaginal specimens for high risk HPV testing. This should lead to increased coverage and marked detection of $\geq$ CIN II/ $\geq$ CIN III lesions, particularly in women who have not attended the previous round of screening. Implementation is likely to pay off immediately in terms of protecting a subset of nonattendees known to be at increased risk of cervical cancer. 


\section{WHAT IS ALREADY KNOWN ON THIS TOPIC}

More than half of cervical carcinomas in countries with population based screening are diagnosed in women not complying with the cervical screening programme

Offering self sampling of cervicovaginal material for screening has been suggested as a way of increasing compliance with screening

\section{WHAT THIS STUDY ADDS}

Offering high risk HPV testing on self sampled cervicovaginal material for HPV testing to nonresponder women results in increased rates of compliance

The yield of $>$ CIN II/ >CIN III in non-responder women is significantly higher than in regular responder women, indicating that this group of women is at increased for cervical cancer

We thank research staff and technicians of the unit of molecular pathology, VU University Medical Center, Amsterdam, for HPV DNA testing and logistics, the cytotechnicians for cytological testing and logistics, and the administrative coworkers and the information technology team of the department of pathology, VU University Medical Center, Amsterdam, for their supportive work. We are grateful for the active participation in the trial of cytology laboratories of the Academic Medical Center, Onze Lieve Vrouwe Gasthuis, Slotervaart Ziekenhuis and HistoPathologisch anatomisch laboratorium (all in Amsterdam) IJsselmeer ziekenhuis (Lelystad), Leids Cytologisch en Pathologisch Laboratorium (Leiden), Medisch Centrum Alkmaar (Alkmaar), Kennemergasthuis (Haarlem), Tergooiziekenhuizen (Blaricum), Spaarne Ziekenhuis (Hoofddorp), Stichting Artsenlaboratorium/trombosedienst (Saltro: Utrecht), Westfries Gasthuis (Hoorn), and Zaans Medisch Centrum (Zaandam). In addition we acknowledge cooperation with local health councils and over 800 general practitioners and their assistants, who contributed to the care of the women involved. We also thank the gynaecologists for their part in this trial and PALGA for retrieving cytological and histological results and screening histories. Contributors: CILMM was the project leader and designed the study with DAMH, FJvanK, PJFS. MG, DAMH, FJvanK, JB, CJLMM, LR, FV, and PJFS drafted the manuscript. MG and JB analysed the data; JB was responsible for the data analyses. LR and JAMB were responsible for database management. DAMH, PJFS, and CJLMM were responsible for HPV DNA testing. MB was responsible for the screening register of the Regional Screening Organisation Database. IS was responsible for communication with gynaecologists. FJvank was responsible for coordination of cytological testing at the individual laboratories. All authors had full access to all of the data in the study and can take responsibility for the integrity of the data and the accuracy of the data analysis, and critically reviewed the manuscript and approved the final version. CJLMM is guarantor

Funding: The study was partly funded by Delphi Bioscience (formerly Pantarhei Devices), Scherpenzeel, Netherlands, and Zeist, Netherlands, who also provided the self sampling kits, and the screening organisation Noord-Holland and Flevoland, and IKA (Comprehensive Cancer Center Amsterdam). Hybrid capture II kits and universal collection medium were provided by Qiagen, Gaithersburg, MD, USA.

Competing interests: All authors have completed the Unified Competing Interest form at www.icmje.org/coi_disclosure.pdf (available on request from the corresponding author) and declare that (1) CJ M, and PJF S have support from Delphi Bioscience B.V. Scherpenzeel, Netherlands, and Qiagen, Gaithersburg, USA, for the submitted work; (2) CJ M, PJ S, and DA $H$ have relationships with Self-screen and CI M has a relationship with Qiagen that might have an interest in the submitted work in the previous 3 years; (3) their spouses, partners, or children have no financial relationships that may be relevant to the submitted work; and (4) none of the authors have non-financial interests that may be relevant to the submitted work. The sources of funding did not have any influence on the design and the analysis of the results.

Ethical approval: This study was approved by the national ethics committee (Ministry of Public Health No 2006/01WBO) and informed consent was given by all women.

Data sharing: No additional data available.

1 Bais AG, van Kemenade FJ, Berkhof J, Verheijen RH, Snijders PJ, Voorhorst $F$, et al. Human papillomavirus testing on self-sampled cervicovaginal brushes: an effective alternative to protect nonresponders in cervical screening programs. Int J Cancer 2007;120:1505-10

2 Raab SS, Grzybicki DM, Zarbo RJ, Jensen C, Geyer SJ, Janosky JE, et al. Frequency and outcome of cervical cancer prevention failures in the United States. Am J Clin Pathol 2007;128:817-24.

3 Sawaya GF, Grimes DA. New technologies in cervical cytology screening: a word of caution. Obstet Gynecol 1999;94:307-10.

4 Sung HY, Kearney KA, Miller M, Kinney W, Sawaya GF, Hiatt RA. Papanicolaou smear history and diagnosis of invasive cervical carcinoma among members of a large prepaid health plan. Cancer 2000;88:2283-9.

5 Peto J, Gilham C, Fletcher O, Matthews FE. The cervical cancer epidemic that screening has prevented in the UK. Lancet 2004;364:249-56.

6 Sasieni P, Adams J, Cuzick J. Benefit of cervical screening at different ages: evidence from the UK audit of screening histories. Br J Cancer 2003:89:88-93

7 Wikstrom I, Stenvall H, Wilander E. Attitudes to self-sampling of vaginal smear for human papilloma virus analysis among women not attending organized cytological screening. Acta Obstet Gynecol Scand 2007;86:720-5.

8 Wright TC Jr, Denny L, Kuhn L, Pollack A, Lorincz A. HPV DNA testing of self-collected vaginal samples compared with cytologic screening to detect cervical cancer. JAMA 2000;283:81-6.

9 Brink AA, Meijer CJ, Wiegerinck MA, Nieboer TE, Kruitwagen RF, van Kemenade $F$, et al. High concordance of results of testing for human papillomavirus in cervicovaginal samples collected by two methods, with comparison of a novel self-sampling device to a conventional endocervical brush. J Clin Microbiol 2006;44:2518-23.

10 Bulk S, van Kemenade F), Rozendaal L, Meijer CJ. The Dutch CISOE-A framework for cytology reporting increases efficacy of screening upon standardisation since 1996. J Clin Pathol 2004;57:388-93.

11 Bulkmans NW, Berkhof I, Rozendaal L, van Kemenade F], Boeke AJ, Bulk S, et al. Human papillomavirus DNA testing for the detection of cenvical intraepithelial neoplasia grade 3 and cancer: 5-year followup of a randomised controlled implementation trial. Lancet 2007:370:1764-72.

12 Hopman EH, Rozendaal L, Voorhorst FJ, Walboomers JM, Kenemans P, Helmerhorst TJ. High risk human papillomavirus in women with normal cervical cytology prior to the development of abnormal cytology and colposcopy. BJOG 2000;107:600-4.

13 Hopman EH, Voorhorst F), Kenemans P, Meyer CJ, Helmerhorst TJ. Observer agreement on interpreting colposcopic images of CIN. Gynecol Oncol 1995;58:206-9.

14 Anderson MC. Premalignant and malignant squamous lesions of the cervix. In: Fox H, Wells M, Haines M, Taylor C, eds. Obstetrical and gynaecological pathology. Churchill Livingstone, 1995:292-7.

15 Wright TC. Precancerous lesions of the cervix. Blaustein's pathology of the female genital tract. 4th ed. Springer Verlag, 1995:248-57.

16 Rebolj M, van Ballegooijen M, Berkers LM, Habbema D. Monitoring a national cancer prevention program: successful changes in cevvical cancer screening in the Netherlands. Int / Cancer 2007;120:806-12.

17 Hanselaar AG. Criteria for organized cervical screening programs. Special emphasis on the Netherlands program. Acta Cytol 2002;46:619-29.

18 Casparie M, Tiebosch AT, Burger G, Blauwgeers $\mathrm{H}$, van de Pol A, van Krieken JH, et al. Pathology databanking and biobanking in the Netherlands, a central role for PALGA, the nationwide histopathology and cytopathology data network and archive. Cell Oncol 2007:29:19-24.

19 Bekkers RLM, Hanselaar AGJM, Melchers WJG, Van Westering RP, Boonstra H, Massuger LFAG. [Wait-and-see policy versus loop excision after two consecutive Pap 2 cervical smears: fewer interventions and an equivalent outcome over time; no real contribution to be expected from high-risk human papillomavirus detection.] Ned Tijdschr Geneeskd 2003;147:302-6.

20 Castle PE, Schiffman M, Burk RD, Wacholder S, Hildesheim A, Herrero R, et al. Restricted cross-reactivity of hybrid capture 2 with nononcogenic human papillomavirus types. Cancer Epidemiol Biomarkers Prev 2002;11:1394-9.

21 Castle PE, Rodriguez AC, Porras C, Herrero R, Schiffman M, Gonzalez P, et al. A comparison of cervical and vaginal human papillomavirus. Sex Transm Dis 2007:34:849-55.

22 Hesselink AT, Bulkmans NW, Berkhof J, Lorincz AT, Meijer CJ, Snijders PJ. Cross-sectional comparison of an automated hybrid capture 2 assay and the consensus GP5+/6+ PCR method in a population-based cervical screening program. / Clin Microbiol 2006;44:3680-5.

23 Karwalajtys T, Howard M, Sellors JW, Kaczorowski J. Vaginal self sampling versus physician cervical sampling for HPV among younger and older women. Sex Transm Infect 2006;82:337-9.

24 Khanna N, Mishra SI, Tian G, Tan MT, Arnold S, Lee C, et al. Human papillomavirus detection in self-collected vaginal specimens and matched clinician-collected cervical specimens. Int J Gynecol Cancer 2007;17:615-22. 
25 Petignat P, Faltin DL, Bruchim I, Tramer MR, Franco EL, Coutlee F. Are self-collected samples comparable to physician-collected cervical specimens for human papillomavirus DNA testing? A systematic review and meta-analysis. Gynecol Oncol 2007;105:530-55.

26 Bulk S, Bulkmans NW, Berkhof J, Rozendaal L, Boeke AJ, Verheijen $\mathrm{RH}$, et al. Risk of high-grade cervical intra-epithelial neoplasia based on cytology and high-risk HPV testing at baseline and at 6-months. Int J Cancer 2007;121:361-7.

27 Bulkmans NW, Rozendaal L, Snijders PJ, Voorhorst F), Boeke AJ, Zandwijken GR, et al. POBASCAM, a population-based randomized controlled trial for implementation of high-risk HPV testing in cervical screening: design, methods and baseline data of 44,102 women. In l Cancer 2004;110:94-101.

28 Overmeer RM, Henken FE, Snijders PJ, Claassen-Kramer D, Berkhof J, Helmerhorst TJ, et al. Association between dense CADM1 promoter methylation and reduced protein expression in high-grade CIN and cervical SCC. J Pathol 2008;215:388-97.

Accepted: 25 January 2010 